\title{
Feasibility of large-scale screening using N-ERC/mesothelin levels in the blood for the early diagnosis of malignant mesothelioma
}

\author{
KOHTA IMASHIMIZU ${ }^{1}$, KAZU SHIOMI ${ }^{1}$, MASAHIRO MAEDA $^{4}$, NAOKO AOKI $^{2,4}$, KIYOKO IGARASHI $^{3}$, \\ FUMIO SUZUKI ${ }^{3}$, MITSURU KOIZUMI ${ }^{5}$, KENJI SUZUKI $^{1}$ and OKIO HINO ${ }^{2,3}$ \\ Departments of ${ }^{1}$ General Thoracic Surgery, Respiratory Medicine, Human Pathology, and ${ }^{2}$ Pathology and Oncology, \\ Juntendo University, School of Medicine, Bunkyo-ku, Tokyo 113-8421; ${ }^{3}$ Environmental Carcinogenesis Research Center, \\ Itabashi-ku, Tokyo 173-0025; ${ }^{4}$ Immuno-Biological Laboratories, Co., Ltd., Fujioka-shi, Gunma 375-0005; \\ ${ }^{5}$ National Institute of Radiological Sciences, Inage-ku, Chiba 263-8555, Japan
}

Received December 16, 2010; Accepted February 28, 2011

DOI: $10.3892 /$ etm.2011.225

\begin{abstract}
A large-scale screening involving the measurement of $\mathrm{N}-\mathrm{ERC} / \mathrm{mes}$ thelin levels in blood using an ELISA system for the early diagnosis of malignant mesothelioma (MM) was carried out in individuals with a history of employment at construction sites. Approximately 30,000 subjects were screened. Of the 80 subjects with high-risk values, one male patient was diagnosed as having MM based on a PET study and histopathology. This is the first report of the pre-clinical diagnosis of MM based on blood test screening. In addition, plasma levels of N-ERC/mesothelin may be effectively used for monitoring relapse after surgery.
\end{abstract}

\section{Introduction}

Mesothelioma is an aggressive tumor arising from the mesothelium, a membrane lining various body cavities, including the pleura, peritoneum and pericardium, and is usually associated with asbestos exposure.

Due to the long incubation period and the short survival time after the onset of asbestos-induced malignant mesothelioma (MM), an early diagnostic system for individuals with a history of asbestos exposure is critically required. Recently, soluble mesothelin-related protein (1) and serum mesothelin have been reported to be potentially useful markers for the early diagnosis of MM $(2,3)$.

A $71-\mathrm{kDa}$ precursor protein of human ERC/mesothelin can be cleaved into a $40-\mathrm{kDa}$ C-terminal fragment as a surface GPI-anchored glycoprotein and a 31-kDa N-terminal fragment as a secreted protein. We focused on this N-ERC/

Correspondence to: Professor Okio Hino, Department of Pathology and Oncology, Juntendo University, School of Medicine, 2-1-1 Hongo, Bunkyo-ku, Tokyo 113-8421, Japan

E-mail: ohino@juntendo.ac.jp

Key words: mesothelioma, mesothelin, ELISA, screening mesothelin, as it is physiologically secreted into the blood, and as a specific ELISA system has been developed for N-ERC/ mesothelin (2,3).

In the present study, to develop a pre-clinical diagnostic system for the early detection of MM, the levels of N-ERC/ mesothelin, the N-terminal 31-kDa fragment of mesothelin, in blood samples was measured by ELISA as a primary screening method. Chest radiography, chest CT and positron emission tomography (PET) examinations, and also histopathology, were then used as secondary screening examinations for individuals with a history of exposure to asbestos and consequently, a high risk of developing of MM.

\section{Materials and methods}

Establishment of ELISA for N-ERC/mesothelin and measurement of blood samples. The sandwich ELISA system used in this report were established as described previously (3). The $7 \mathrm{E} 7 \mathrm{MoAb}$ was used as the capture antibody and the $16 \mathrm{~K} 16$ $\mathrm{MoAb}$ was used as the detecting antibody after conjugation with horseradish peroxidase. Recombinant N-ERC/mesothelin was used as the standard. The protein in the ELISA system was purified from culture supernatants of $\mathrm{CHO}-\mathrm{K} 1$ cells transfected with ERC/mesothelin cDNA using an anti-ERC/ mesothelin PoAb. EDTA plasma was used for this ELISA.

The present study was approved by the Institutional Review Board of Juntendo University School of Medicine, its hospital and Immuno-Biological Laboratories. Written informed consent for participation in the study was obtained from all of the subjects.

\section{Results}

Case reports of three patients

Patient A: clinical history. The subject was a 71-year-old male who had a history of smoking for 58 years. He did not have any clinical complaints or significant past medical history. The patient had an occupational history of employment as a plumber using asbestos for pipe insulation since the age of 24 years. 
History of present illness. The subject underwent a physical examination in June 2007, at which time his plasma N-ERC/ mesothelin level was $8.62 \mathrm{ng} / \mathrm{ml}$, which was higher than the cutoff value (3).

He subsequently underwent secondary screening. Although a chest CT revealed bilateral pleural thickening with calcification, no abnormal findings in the abdominal area or interior of the pelvis were observed in an examination performed in October 2007.

In January 2008, a thoracoabdominal CT revealed an anterior mediastinal node and a small amount of pleural effusion, and the amount of ascites was noted to have increased. In May 2008, a PET-CT examination was performed and an accumulation was detected in the anterior mediastinal node and abdominal cavity (Fig. 1). A laparoscopic biopsy was performed in June 2008 and numerous white nodes were detected in the abdominal cavity. A pathological examination confirmed the diagnosis of MM, epithelioid-type (Fig. 2).

During this time period, the patient's blood N-ERC/ mesothelin levels increased from $8.62 \mathrm{ng} / \mathrm{ml}$ in June 2007 to $13.59 \mathrm{ng} / \mathrm{ml}$ in October 2007 and 31.49 ng/ml in April 2008.

Patient B: clinical history. The subject was a 68 -year-old male. He had suffered from glaucoma since the age of 42 , gout since the age of 50 and high blood pressure and hyperlipidemia since the age of 55 years.He had an occupational history of employment as a quality control supervisor in charge of electric machinery that was stored in heat-insulating material (asbestos) in his 20s, in an electrical construction company where spraying with asbestos was carried out in his immediate environment in his 30s, and in a building custodial service using asbestos for caulking projects around pumps from the age of 40 .

History of present illness. The subject underwent a physical examination each year. In 2007, an abnormality was detected on a plain chest roentgenogram. In November 2008, an abnormality was again perceived on a plain chest roentgenogram. In December 2008, a pleural effusion was detected at a local hospital. In February 2009, thoracentesis was performed, and a cytological examination of the pleural fluid revealed class $\mathrm{V}$ cytology.

The patient subsequently visited the clinic of Juntendo University Hospital in the same month, at which time his plasma N-ERC/mesothelin level was $15.6 \mathrm{ng} / \mathrm{ml}$, which was in excess of the cutoff value (3). In April 2009, a pleural biopsy was performed. Histopathological examination confirmed a diagnosis of MM, epithelioid-type. The TNM class was evaluated as pT1bNOM0 and the clinical stage as stage IB. In June, the patient was admitted to the hospital. Surgical removal of the pericardium and diaphragm and left extrapleural pneumonectomy were performed immediately. On day 3 after the surgery, the thoracostomy tube was removed. On day 11, as he was making satisfactory progress, he was discharged from the hospital, at which time his plasma N-ERC/mesothelin level was $1.63 \mathrm{ng} / \mathrm{ml}$, lower than the cutoff value. After discharge from the hospital, he visited the clinic again in June, July, August and December 2009, and February 2010, and no evidence of recurrence was detected. In December 2009, the plasma N-ERC/mesothelin level was $2.64 \mathrm{ng} / \mathrm{ml}$, which was within the normal range.

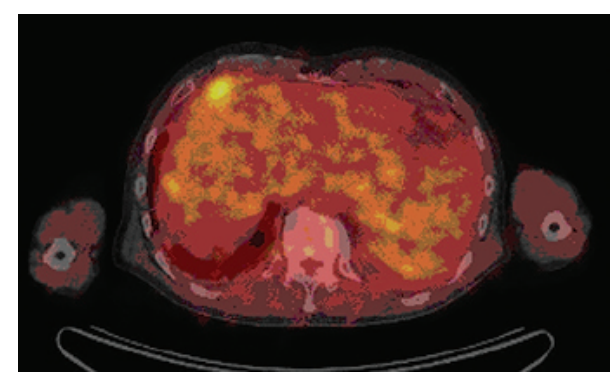

Figure 1. FDG PET/CT scan shows an 18x12-mm nodule located between the diaphragm and liver. It displayed strong FDG tracer uptake.

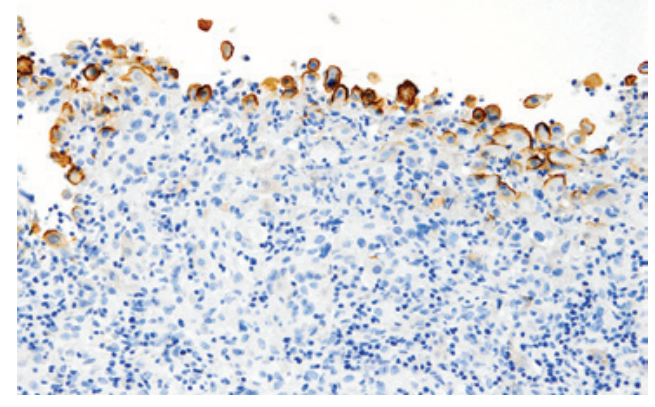

Figure 2. Tumor biopsy from the peritoneum shows mesothelioma, epithelioid-type. Mesothelioma cells exhibited ERC/mesothelin expression on the outer surface.

Patient C: clinical history. The subject was a 68-year-old female. She had a previous history of undergoing surgical removal of a chocolate cyst in the right ovary. She had an occupational history of employment as a teacher in a school building with asbestos insulation.

History of present illness. The patient visited a local hospital with the chief complaint of dyspnea on exertion and was diagnosed as having a bloody pleural effusion on the left side.

A tumor mass on the chest wall was detected by chest CT. A lung biopsy was performed. Cytological examination of the pleural fluid revealed class III cytology, and biochemical examination revealed a markedly elevated level of hyaluronic acid $(646,000 \mathrm{ng} / \mathrm{ml})$. Cytological examination of the lacteal gland discharge revealed class V cytology. In April 2009, the patient visited the clinic of Juntendo University Hospital. A pleural biopsy was performed. Histopathological examination confirmed the diagnosis of malignant pleural mesothelioma. The TNM classification was evaluated as pT4N2M0 and the clinical stage as IV. She was discharged from the hospital, but was re-admitted in May of the same year because of re-accumulation of the pleural fluid, which was treated by drainage. A pleurosclerosis was performed using OK-432 and 5KE. She was discharged from the hospital, but was again admitted in June 2009, at which time her plasma N-ERC/mesothelin level reached $117.50 \mathrm{ng} / \mathrm{ml}$, markedly higher than the cutoff value. A left extrapleural pneumonectomy was performed. On day 3 after the surgery, the thoracostomy tube was removed. On day 9, as she was making satisfactory progress, she was discharged from the hospital. After the surgery, her plasma N-ERC/mesothelin levels were $14.46 \mathrm{ng} / \mathrm{ml}$ in December 2009, $7.13 \mathrm{ng} / \mathrm{ml}$ 
in February 2010 and $8.36 \mathrm{ng} / \mathrm{ml}$ in April 2010. She showed no evidence of recurrence until February 2010. In July 2010 , she was found to have a tumor recurrence on the chest wall and a metastasis in the contralateral lung, and was re-admitted to the hospital for chemotherapy, at which time her plasma $\mathrm{N}-\mathrm{ERC} / \mathrm{mesothelin}$ level had again increased to $36.03 \mathrm{ng} / \mathrm{ml}$.

Relationship between the plasma level of N-ERC/mesothelin and the pathogenesis of MM. Patient A was detected during a large-scale screening of approximately 30,000 subjects affiliated with the Tokyo Doken National Health Insurance Association who had worked at construction sites, and who had undergone a physical examination and submitted blood samples for laboratory analysis. The N-ERC/mesothelin level in each blood sample was measured using our novel ELISA method.

Approximately 80 subjects were identified as belonging to a high-risk group based on the previously reported cutoff value for $\mathrm{N}$-ERC/mesothelin (3). Of these subjects, 30 individuals underwent secondary screening, and one man (patient A) was diagnosed as having MM based on the results of a PET study and histopathology. It is noteworthy that, in this case, although no abnormal findings were observed on a thoracoabdominal CT performed in October 2007, the plasma N-ERC/ mesothelin level was already elevated at this time. Thus, this is the first report of the pre-clinical diagnosis of MM based on a blood test.

The two additional cases reported here were not detected to have MM in the large-scale screening study, but they represent appropriate examples to demonstrate the effectiveness of $\mathrm{N}-\mathrm{ERC} / \mathrm{mes}$ thelin as a blood marker for the diagnosis of MM. Patient B was a 68-year-old male who was detected to have an abnormality on a plain chest X-ray during a medical check-up. A histopathological examination confirmed the diagnosis of MM, epithelioid-type. Surgical removal of the pericardium and diaphragm and a left extrapleural pneumonectomy were performed. Before the surgery, the plasma N-ERC/mesothelin level was $15.6 \mathrm{ng} / \mathrm{ml}$; however, following the surgery it decreased to $1.63 \mathrm{ng} / \mathrm{ml}$. There has been no evidence of recurrence since.

Patient $\mathrm{C}$ was a 68-year-old female working as a teacher in a school building with asbestos insulation. She had a previous history of surgical removal of a chocolate cyst in the right ovary. She visited our hospital because of dyspnea on exertion, and was diagnosed as having a bloody pleural effusion on the left side. Histopathologic examination of a pleural biopsy confirmed the diagnosis of malignant pleural mesothelioma. A left extrapleural pneumonectomy was performed. Before the surgery, the plasma N-ERC/mesothelin level was $117.50 \mathrm{ng} /$ $\mathrm{ml}$, while after the surgery it decreased to $7.13 \mathrm{ng} / \mathrm{ml}$. However, 11 months later, the patient developed a tumor recurrence on the chest wall and a metastasis in the contralateral lung, at which time her plasma $\mathrm{N}-\mathrm{ERC} /$ mesothelin level had again increased to $36.03 \mathrm{ng} / \mathrm{ml}$.

As described above, the plasma level of N-ERC/mesothelin may be a useful blood marker for the early diagnosis of MM, and may be particularly effective for monitoring patients for relapse after surgery.

Early diagnosis of MM may become possible by measurement of the blood $\mathrm{N}-\mathrm{ERC} / \mathrm{mes}$ thelin level as a routine laboratory test in high-risk populations with a history of exposure to asbestos.

\section{Discussion}

This study showed the effectiveness of N-ERC/mesothelin as a blood marker for MM. At present, two lines of clinical studies for mesothelin-bearing tumors are ongoing: a phase I trial of the anti-mesothelin immunotoxin SS1P in patients with mesothelioma, ovarian and pancreatic cancer (4), and a phase I clinical trial of MORAb-009, which is a chimeric antibody targeting tumor-associated mesothelin $(5,6)$. Additionally, Inami et al reported the antitumor activity of the anti-C-ERC/mesothelin monoclonal antibody in vivo (7), and Yoshida et al reported on the use of PET imaging of 64Cu-labeled Fab for detecting ERC/ mesothelin in a mesothelioma mouse model (8).

It would be most useful to combine immunotherapy using an anti-mesothelin antibody directed against mesothelinbearing tumors and a blood test for ERC/mesothelin.

The screening protocol reported herein is very promising for the early diagnosis of MM in subjects with a history of exposure to asbestos. In addition, measurement of the plasma level of N-ERC/mesothelin may also be useful in the follow-up of patients after surgery for MM.

\section{Acknowledgements}

This study was supported by a Grant-in-Aid for Cancer Research and Grants-in Aid for Scientific Research from the Ministry of Education, Culture, Sports, Science, and Technology of Japan, and the Ministry of Health, Labor, and Welfare of Japan.

\section{References}

1. Robinson BWS, Creaney J, Lake R, Nowak A, Musk AW and De Klerk N: Mesothelin-family proteins and diagnosis of mesothelioma. Lancet 362: 1612-1616, 2003.

2. Shiomi K, Miyamoto H, Segawa T, Hagiwara Y, Ota A and Hino O: Novel ELISA system for detection of NERC/mesothelin in the sera of mesothelioma patients. Cancer Sci 97: 928-932, 2006.

3. Shiomi K, Hagiwara Y, Sonoue K, et al: Sensitive and specific new enzyme-linked immunosorbent assay for N-ERC/mesothelin increases its potential as a useful serum tumor marker for mesothelioma. Clin Cancer Res 14: 1431-1437, 2008.

4. Hassan R, Bullock S, Premkumar A, Kreitman RJ, Kindler H, Willingham M and Pastan I: Phase I study of SS1P, a recombinant anti-mesothelin immunotoxin for targeted therapy of mesothelin-expressing mesotheliomas, ovarian and pancreatic cancer. J Clin Oncol 25: 3553, 2007.

5. Hassan R, Ebel W, Routhier E, et al: Preclinical evaluation of MORAb-009, a chimeric antibody targeting tumor-associated mesothelin. Cancer Immun 7: 20-29, 2007.

6. Hassan R and Ho M: Mesothelin-targeted cancer immunotherapy. Eur J Cancer 44: 46-53, 2008.

7. Inami K, Abe M, Takeda K, Hagiwara Y, Maeda M, Segawa T, Suyama M, Watanabe S and Hino O: Antitumor activity of anti$\mathrm{C}$-ERC/mesothelin monoclonal antibody in vivo. Cancer Sci 101: 969-974, 2010.

8. Yoshida C, Sogawa C, Tsuji A, Sudo H, Sugyo A, Uehara T, Hino O, Yoshii Y, Fujibayashi Y, Fukumura T, Koizumi M, Arano $\mathrm{Y}$ and Saga T: Development of positron emission tomography imaging by $64 \mathrm{Cu}$-labeled $\mathrm{Fab}$ for detecting ERC/ mesothelin in mesothelioma mouse model. Nucl Med Commun 31: 380-388, 2010. 